\title{
Evaluación del modo lingüístico escribir con posibilidad de revisión y corrección del texto
} $\cdots$
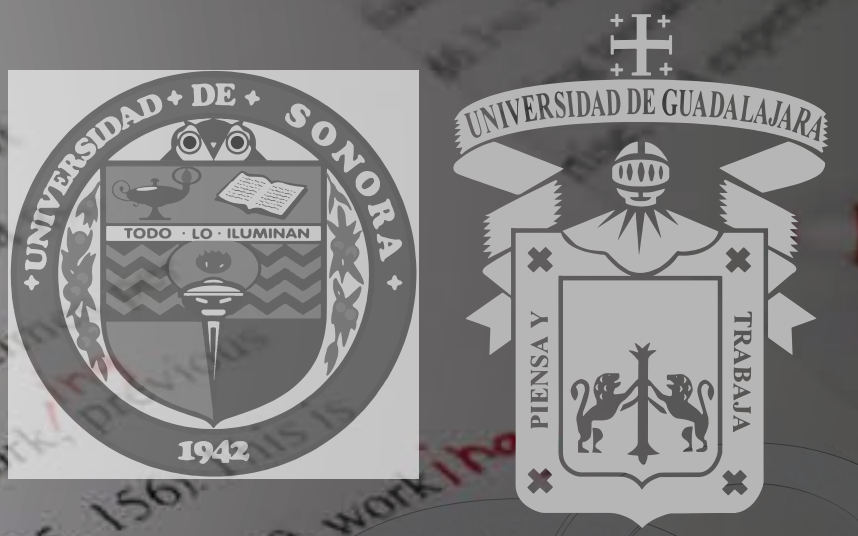
ar

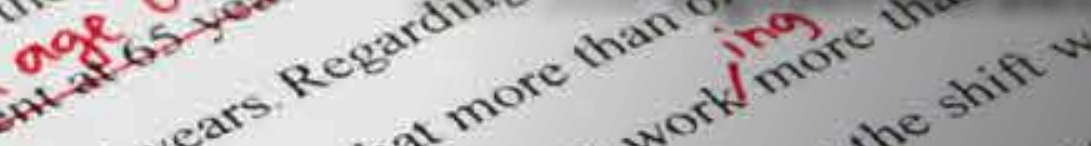
150

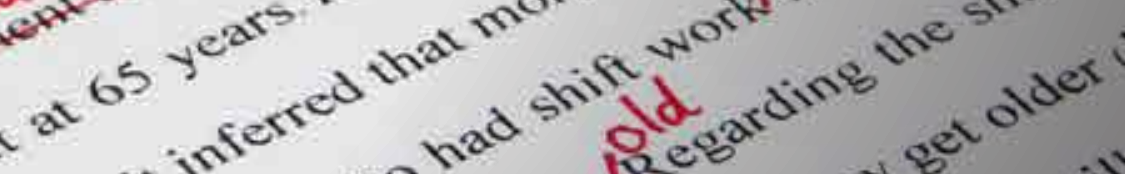

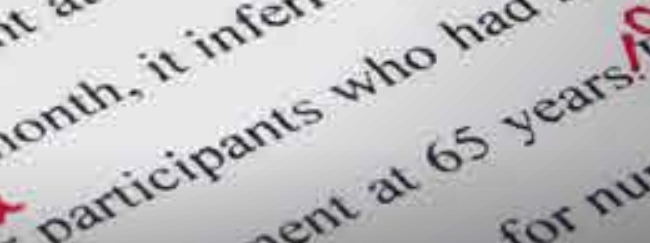


ID:

\subsection{1/2027-1786.RIP.12106}

Title: Evaluation of the linguistic way of writing with the possibility of revision and correction of the text

Título: Evaluación del modo lingüístico escribir con posibilidad de revisión y corrección del texto

Alt Title / Título alternativo:

[en]: Evaluation of the linguistic way of writing with the possibility of revision and correction of the text

[es]: $\quad$ Evaluación del modo lingüístico escribir con posibilidad de revisión y corrección del texto

Author (s) / Autor (es):

López Corral, Acuña Meléndrez, Flores Aguirre, \& Irigoyen Morales

Keywords / Palabras Clave:

[en]: enabling, linguistic modes, writing, revision, correction

[es]: habilitación, modos lingüísticos, escritura, revisión, corrección

\section{Resumen}

En investigaciones sobre escritura se ha señalado que revisary corregir los textos son aspectos fundamentales cuando se escribe. Se considera también que la manera en que escriben las personas puede estudiarse por medio de la habilitación lingüística, definida

como la facilitación de un desempeño en modo lingüístico activo (señalar, escribir o hablar) como resultado de la exposición previa

a un modo lingüístico reactivo (observar,

leer o escuchar). Por ello, el objetivo de este estudio fue evaluar la exposición de estudiantes universitarios a los modos reactivos, sobre la habilitación lingüística del modo activo escribir y sobre la revisión y corrección cuando se les presenta de nueva cuenta el texto escrito. Los participantes, asignados a uno de tres grupos, primero escribieron un texto sobre el ciclo del agua, después fueron expuestos a uno de los tres modos lingüísticos reactivos, por último, se les presentó el texto escrito previamente sobre el ciclo del agua para su revisión y corrección. Los resultados mostraron puntajes de aciertos más altos para los grupos expuestos a los modos

leer y escuchar, seguidos por el grupo donde debían observar. No se encontró corrección de los textos. Se discuten los resultados en relación con investigaciones de habilitación lingüística y con aspectos necesarios para la revisión y corrección de textos.

\section{Abstract}

In research about writing, it's been indicated that revision and correction of text are fundamental aspects of writing. It's also suggested that the way people write can be studied based on linguistic modes, defined as facilitation of the performance in an active linguistic mode (indicating, writing or talking) as a result of the previous exposure to a reactive linguistic mode (observing, reading or listening). The purpose of this study was

to evaluate the effect of the exposure of undergraduates to different reactive modeson

the linguistic mode of writing, as well as revision and correction when the written text is presented to them again. The participants, assigned to one of three groups, first wrote a text about the water cycle, then were exposed to one of three reactive linguistic modes, and

last, were presented with the text that they had previously written about the water cycle to check and correct. The results show higher scoresin the groups exposed to the reading and listening modes, followed by those that were supposed to observe; no correction on the texts was found. The results are discussed in terms of linguistic modes research and the required aspects of revision and correction of texts interventions in mental and physical health issues based on the recognition of diversity and interculturality

Submited:

2018-10-09

Acepted:

2019-01-11

\section{Citar como:}

López Corral, A., Acuña Meléndrez, K. F., Flores Aguirre, C. J., \& Irigoyen Morales, J. J. (2019). Evaluación del modo lingüístico escribir con posibilidad de revisión y corrección del texto. Revista Iberoamericana de Psicología, 12 (1), 61-76. Obtenido de: https://reviberopsicologia.ibero.edu.co/article/view/rip.12106

Alfonso López Corral, Msc Psi

ORCID:

\section{0-0003-2557-808X}

Source | Filiacion:

Universidad de Sonora

BIO:

Profesor Auxiliar de la Licenciatura en Psicología del Instituto Tecnológico de Sonora y empleado Administrativo de la Universidad de Sonora

City | Ciudad:

Navojoa [mx]

e-mail:

alfonso.lopez@unison.mx
Karla Fabiola Acuña Meléndrez, Msc Psi

ORCID:

0000-0002-1704-4240

Source | Filiacion:

Universidad de Sonora

BIO:

Investigadora SNI Nivel I. Profesora de Tiempo Completo de la Licenciatura en Psicología del Departamento de Psicología y Ciencias de la Comunicación de la Universidad de Sonora.

\section{City | Ciudad:}

Hermosillo [mx]

e-mail:

karla.acuna@uson.mx
Carlos Javier Flores Aguirre, MA PS

AutorID: ID: 7103184899 ORCID: $\quad$ 0000-0002-3085-4029

Source | Filiacion:

Universidad de Guadalajara

BIO:

Investigador SNI Nivel II. Profesor Investigador del Centro de Estudios e Investigaciones en Comportamiento CEIC, de la Universidad de Guadalajara.

City | Ciudad:

Guadalajara [mx]

e-mail:

carlos.faguirre@academicos.udg.mx
Juan José Irigoyen Morales, Msc Psi

ORCID:

0000-0002-7098-0388

Source | Filiacion:

Universidad de Sonora

BIO:

Investigador SNI Nivel I. Profesor de Tiempo Completo de la Licenciatura en Psicología del Departamento de Psicología y Ciencias de la Comunicación de la Universidad de Sonora.

City | Ciudad:

Hermosillo [mx]

e-mail:

irigoyen@psicom.uson.mx 


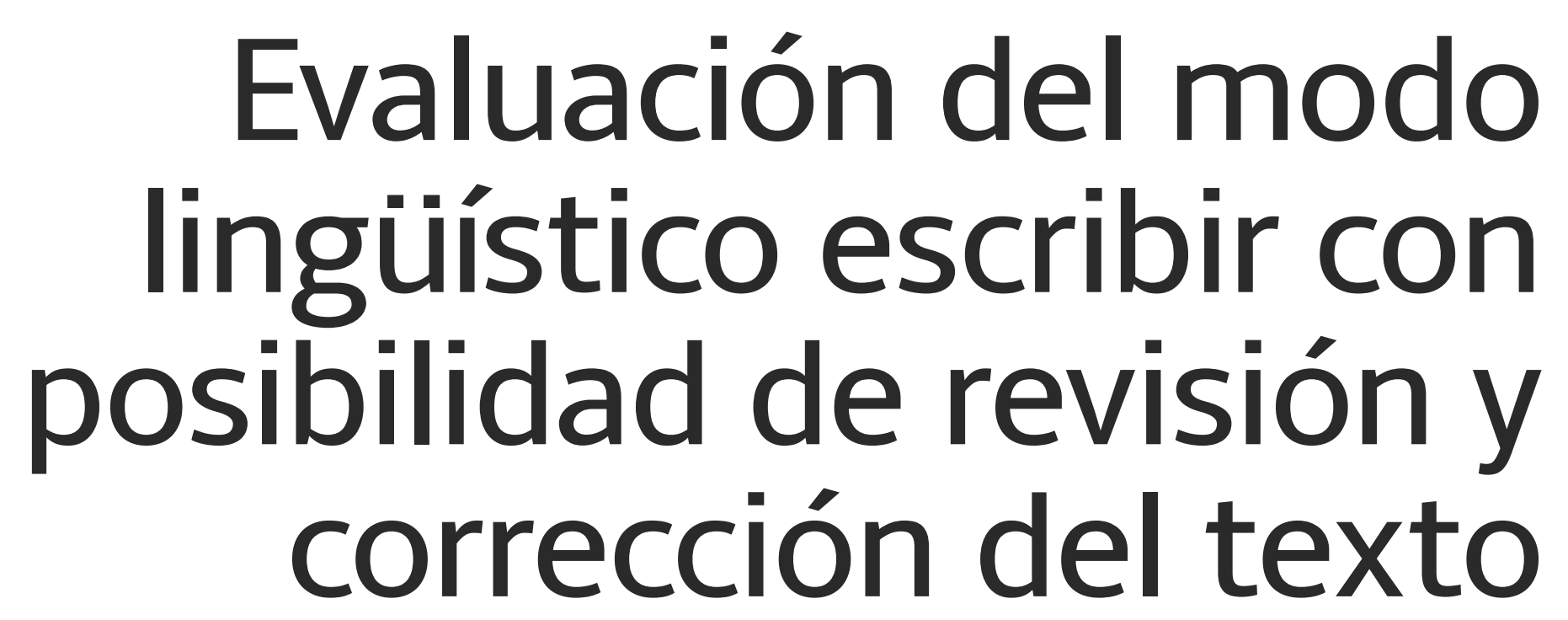

Evaluation of the linguistic way of writing with the possibility of revision and correction of the text

\author{
Alfonso López Corral \\ Karla Fabiola Acuña Meléndrez \\ Carlos Javier Flores Aguirre \\ Juan José Irigoyen Morales
}

A partir del supuesto de que escribir es un comportamiento lingüístico, se busca una caracterización del escribir que permita identificar las ejecuciones por la mediación que el escritor realiza de los referentes sobre los que escribe y por la trasformación que realiza del texto a partir de estos mismos referentes, y no por la determinación $a$ priori basada en los tipos de textos que se escriben. Esto obedece también al interés de estudiar la escritura de una manera directa, esto es, el contacto entre el escritor y su referente, es decir, el contacto entre el escritor y aquello de lo que escribe. Para ello, se hace el análisis de la escritura a partir de los llamados modos lingüísticos (Fuentes \& Ribes, 2001) es decir, las diferentes formas en las que el lenguaje ocurre. Una idea base es que el texto que un escritor elabora, a través del contacto con un referente, no será el mismo si este se presenta de diferentes, es decir, no será lo mismo si se observa, escucha o lee sobre aquello que habrá de escribirse. Respecto a la situación de escritura, es igual de importante el papel del escritor como su propio lector, porque es lo que se considera que posibilita finalmente la revisión y corrección, que son aspectos cruciales de la escritura. En esta línea,Tamayo, Ribes \& Padilla (2010) propusieron el estudio de las relaciones entre los modos lingüísticos mediante la investigación de lo que llamaron habilitación lingüística, que hace referencia a cómo se habla, se gesticula o señala y a cómo se escribe, después de leer, observar o escuchar algo. Es a partir de esta noción que se consideró que podría estudiarse el contacto que el escritor realiza con sus referentes, en breve, estudiar cómo el escritor se habilita para escribir. 


\section{Marco teórico}

Desde una perspectiva psicológica orientada al estudio del comportamiento, escribir puede considerarse como un modo lingüístico. Los modos lingüísticos refieren a la forma en que las personas se comportan lingüísticamente, es decir, cómo escuchan, hablan, leen, escriben, observan y señalan. (Fuentes \& Ribes, 2001). Los autores ligados a esta interpretación del escribir explican que estos tipos de conducta lingüística dependen del medio físico y del sistema reactivo del individuo, así como de las combinaciones apropiadas de éstos. La adaptación de cada modo lingüístico implica los sistemas reactivos visual, auditivo, fonador, motor grueso, motor manual y digital fino; así como los medios de ocurrencia óptico y acústico (Camacho \& Gomez, 2007) Por ejemplo, escribir se ejercita mediante respuestas motoras (movimientos que con la práctica se van afinando y volviendo precisos) con consecuencias visuales en la forma de trazos impresos de carácter específico, esto es, símbolos y signos específicos; por su parte, leer ocurre como fonación explícita en correspondencia a grafemas particulares (Gomez, 2005). Fuentes \& Ribes (2001) agregan que los modos tienen lugar como pares complementarios y pueden agruparse en activos y reactivos. Dentro de los modos activos se tienen las conductas de gesticular/señalar, hablar y escribir. A su vez, dentro de los reactivos ubicaríamos las conductas de observar, escuchar y leer. De acuerdo con esto, los pares complementarios se constituyen de la siguiente forma: a observar corresponde gesticular/señalar, a escuchar corresponde hablary a leer corresponde escribir.

A partir de esta concepción se han impulsado investigaciones que proporcionan datos sobre el establecimiento y desarrollo de los modos lingüísticos como variables críticas en el estudio del lenguaje desde una perspectiva psicológica; (Camacho, Irigoyen, Gomez, Jimenez, \& Acuña, 2007; Fuentes \& Ribes, 2001) pero también sobre fenómenos de especial interés para la ciencia de la conducta, como lo son el aprendizaje y la comprensión (Acuña, Irigoyen, \& Jimenez, 2013; Quiroga, Peña, \& Padilla, 2013). Por ejemplo, Acuña et al. (2013) Llevaron a cabo tres estudios para evaluar la comprensión de contenidos científicos a partir de los modos lingüísticos escribir y hablar. El objetivo fue analizar si el explicitación del criterio de ajuste, las variaciones en el tipo de tarea, su nivel funcional y el modo lingüístico involucrado, determinaban ajustes diferenciales en la comprensión de un material presentado en texto o en audio que correspondía a contenidos de carácter científico. La situación de evaluación se conformó por la presentación de dicho material en texto y audio, una prueba de diez reactivos de opción múltiple y diez de respuesta abierta, así como por las tareas de elaborar un resumen y una conclusión.

Los resultados mostraron que el desempeño de los participantes fue diferencial, en el sentido de que aquellas tareas consideradas como menos complejas (prueba de opción múltiple y resumen) fueron las que presentaron mayor porcentaje de aciertos; también que el nivel de ejecución fue mayor (aproximadamente en un $20 \%$ en los tres estudios) en la modalidad textual del objeto referente que en la auditiva; que las variaciones en las secuencias de presentación del modo lingüístico mostraron diferencias en términos de mayor porcentaje de congruencia si primero se escribía y después se hablaba, en comparación a si primero hablaban y después escribían. Con base en esto, los autores destacaron que el explicitación del criterio de ajuste tiene un efecto facilitador sobre la comprensión de contenidos científicos, y que el desempeño de los participantes pareció depender más del modo lingüístico, del tipo de tarea y su nivel de complejidad, que del contenido de los materiales de estudio. En este sentido, hay dos supuestos teóricos que le dan sustento a estos hallazgos: 1) que los modos lingüísticos tienen propiedades funcionales particulares que modulan diferencialmente la interacción del individuo con el medio, pero que también esto dependerá del tipo de tarea y su complejidad. Por ejemplo, escribir puede ser tan simple o tan complejo según se requiera en el tipo de tarea; 2) se asume que los modos reactivos y activos son funcionalmente complementarios y que dicha complementariedad depende del desarrollo de cada modo dentro de dominios particulares.

La relevancia de la investigación de los modos lingüísticos puede inferirse a partir de lo que señalaron Camacho y Gómez (2007) en el sentido de que es evidente la diferencia entre adquirir conocimiento señalando las características de lo que se aprende, hablando sobre lo que se aprende o generando un texto sobre el mismo. En el estudio de los modos lingüísticos comenzó a investigarse la habilitación lingüística. Tamayo et al., (2010) definieron la habilitación lingüística como la facilitación de un desempeño en un modo lingüístico activo como resultado de la exposición a un modo lingüístico reactivo. La habilitación lingüística sería entonces la manera en que el leer, escuchar u observar afecta el desempeño al momento de escribir, hablar o señalar/gesticular.

Las investigaciones en habilitación lingüística se han realizado por medio del procedimiento de igualación de la muestra de primer y segundo orden, aunque no exclusivamente (Pulido \& Tamayo, 2015). Con el propósito de observar cuál modo reactivo habilitaba de una manera más efectiva los modos activos, Tamayo et al. (2010) Tamayo y Martínez (2014) Por medio del procedimiento de igualación de la muestra de primer orden y segundo orden, evaluaron la habilitación lingüística de la respuesta de escribir, hablar y señalar, al exponer a los participantes a los modos reactivos leer, observar y escuchar. En ambos estudios utilizaron figuras geométricas como estímulos que, de acuerdo al modo correspondiente, se presentaban para que se escucharan (por ejemplo, un audio reproducía la palabra triángulo), leyeran (se mostraba escrita la palabra triángulo) u observaran (se presentaba la figura geométrica del triángulo). Los participantes debían escribir el nombre del estímulo de comparación (ECO), decir (hablar) el nombre del ECO o señalar el ECO que consideraran correcto.

Los autores reportaron un mayor porcentaje de respuestas correctas del modo escribir cuando el modo reactivo fue observar (Tamayo, Ribes, \&Padilla, 2010) y delosparesobservar-señalaryobservarescribir, seguidos por los pares leer-hablar y leer señalar(Tamayo \& Martinez, 2014). En ambos estudios el modo observar se ubicó en los porcentajes más altos de respuestas correctas. Al respecto, Pulido \&Tamayo (2015) sugirieron que los elevados porcentajes de respuestas correctas pudieron deberse al tipo de tarea empleada, así como a las características y propiedades de los estímulos, dadas las características perceptuales de la tarea (igualación a la muestra) y el tipo de estímulos utilizados (figuras geométricas).

Se considera que la noción de habilitación lingüística permite abordar algunos elementos constitutivos de la escritura a partir de su concepción como comportamiento lingüístico y que permite identificar las ejecuciones sin determinarlas a priori por los tipos de textos que se escriben sino por la mediación que el escritor realiza de los referentes sobre los que escribe y por la trasformación que realiza del texto a partir de estos mismos referentes. Esta caracterización obedece también al interés de estudiar la escritura de una manera directa, esto es, el contacto entre el escritor y su referente. Una idea base es que el texto que un escritor elabora a través del contacto con un referente no será el mismo si este se presenta de diferentes formas, es decir, que no será lo mismo si se observa, escucha o lee sobre aquello que habrá de escribirse. 
Teniendo como base los estudios de habilitación lingüística, pero también estas consideraciones sobre la escritura, López, Flores \&Torres (2017) evaluaron la habilitación lingüística del modo activo escribir, con la diferencia de que no utilizaron el procedimiento de igualación de la muestra y se buscó que la respuesta escrita evaluada no tuviera límite de extensión. El supuesto fue que el estudio de la habilitación lingüística permite estudiar la escritura, así como caracterizar la forma en que las personas hacen contacto con el referente sobre el que escriben y, por ende, la posibilidad de analizar el modo lingüístico activo y reactivo correspondiente.15 participantes divididos en tres grupos (Grupo Audio, Grupo Texto y Grupo Video, para su identificación), en la primera fase escribieron de manera libre sobre un tema indicado; en la segunda fase fueron expuestos a uno de los tres modos lingüísticos reactivos: el Grupo Audio debía escuchar una grabación sobre el ciclo del agua, el Grupo Texto leer un texto sobre el ciclo del agua y el Grupo Video observar un video que representaba el ciclo del agua; por último, en la tercera fase debían volver a escribir sobre el tema escrito en la primera fase o pre prueba.

Los resultados mostraron, de acuerdo con las categorías evaluadas, que midieron tanto aspectos de forma como de contenido (por ejemplo, mención de conceptos o bien uso de conceptos de forma pertinente, entre otros), que los participantes que debían leer o escuchar obtuvieron puntajes más altos en la post prueba en comparación con la pre prueba; en cambio, los participantes que debían observar no mostraron ningún cambio o efecto en la post prueba. Sin embargo, aunque se encontró un aumento en aspectos de forma de manera general, el contenido de los mismos no mostró cambios destacables en los tres grupos. Uno de los aspectos señalados fue que las instrucciones otorgadas a los participantes, al momento de ser expuestos a los modos lingüísticos reactivos, no explicitaban la función que tendrían los mismos, es decir, lo que tendrían que hacer después de escuchar, leer y observar.

Con base en estos resultados, López, Flores e Irigoyen (2018) realizaron una réplica del estudio. De igual manera, 15 participantes fueron asignados a tres grupos con la misma identificación y expuestos a idénticas condiciones que en el estudio antecedente, la única diferencia fue que se les instruyó de manera explícita respecto a la función del material al que estaban por ser expuestos en la fase dos y lo que habrían de hacer a continuación con el mismo. En los resultados, las ejecuciones más altas se ubicaron en los aspectos llamados de forma (en este caso las categorías de frases formuladas y mención de conceptos) y las ejecuciones más bajas en aspectos de contenido (las categorías de instancias descriptivas usadas en las frases, frases referidas a relaciones y conceptos usados de manera pertinente). Al parecer, la exposición al material provocó un aumento de la extensión de la escritura, pero no la precisión de su contenido, la cual se asume que se alcanza mediante práctica, retroalimentación y corrección.

Uno de los aspectos discutidos por los autores fue que los resultados en los dos estudios se vieron afectados porque los participantes debían escribir dos textos en lugar de uno, aunque se escribía sobre lo mismo; es decir, se les negaba la oportunidad de revisar y reescribir lo escrito. Esto es importante, porque como se sabe, una de las particularidades de la escritura es que generalmente se extiende en tiempo (y espacio). Su característica de producto relativamente permanente posibilita que se corrija lo que se escribió, porque permite leerlo y releerlo no necesariamente después de haberlo producido. Además, un supuesto es que sólo se le domina mediante la práctica constante en dominios específicos.

Revisar un texto es hacer contacto de nueva cuenta con un documento escrito. La revisión implica su relectura parcial o completa, la cual se hace mientras se escribe o una vez finalizado el escrito y transcurrido un tiempo variable. La corrección implica la revisión y la modificación parcial o total del documento. La modificación puede hacerse sobre aspectos de forma (cambios en ortografía, gramática, sintaxis; en el ordenamiento y disposición de lo escrito en el cuerpo del documento) o bien sobre contenido (las ideas expuestas que constituyen el texto, ya sea en su ordenamiento o incluso en su reformulación).

Distintos autores han señalado que la revisión y corrección forman parte fundamental de la escritura (Beaugrande, 1987; Cassany, 2010; Flower); pero que ésta no necesariamente se pone en práctica porque hacerlo exige habilidades o competencias que se adquieren mediante el ejercicio constante (Bereiter \& Scardamalia, 1987; Truscott \& Hsu, 2008) y, como ejemplifican Kiuhara, Graham \& Hawken (2009) la realización de tareas en el aula que tienen que ver con escritura suelen ser de baja frecuencia.

El estudio de la revisión y corrección de la escritura se ha identificado principalmente a partir de la diferencia entre lo que hacen escritores expertos y novatos, de acuerdo con lo planteado por Hayes y Flower (1980)en su modelo cognitivo de escritura y por Bereiter y Scardamalia (1987) en sus dos modelos de escritura, Decir el conocimiento y Transformar el conocimiento. Pero también por autores como Casanny (2010), Zimmerman \& Kitsantas (2002), entre otros, que señalan características específicas referidas a estos tipos de escritores. Por ejemplo, de manera general, los escritores expertos al revisar y corregir realizan modificaciones globales (tanto de forma como de contenido) en tanto que los escritores novatos suelen concentrarse sólo en aspectos de forma.

Ahora bien, no todos los trabajos se restringen a evaluar la revisión y corrección bajo el supuesto de lo que hacen escritores expertos y novatos. Algunos se han enfocado a estudiar revisión y corrección y sus efectos sobre el dominio de habilidades de escritura. Se ha investigado, por ejemplo, si la revisión y corrección tiene efectos a largo plazo en participantes que recibieron indicación de sus errores y se les pidió que los corrigieran, encontrando que éstos redujeron la comisión de errores de forma en escritos posteriores, pero no ocurrió lo mismo en la exposición de los contenidos en el texto (Truscott \& Hsu, 2008)

Otros estudios han investigado si el modelamiento y la retroalimentación sobre la corrección favorece la mejora de las habilidades de escritura (Zimmerman \& Kitsantas, 2002), encontrando efectos positivos en aspectos como identificación y corrección de errores, añadido de información necesaria y adecuación a objetivos de escritura en aquellos participantes que observaron e imitaron a un modelo experto mientras escribía, contrario a quienes no contaron con un modelo; o bien se ha estudiado si el señalamiento de errores es efectivo en la mejora de los textos (Hyland, 203) encontrando que aquellos participantes que reciben retroalimentación sobre sus errores mejoran en la precisión de los contenidos respecto a los temas asignados para escribir, contrario a los que no reciben dicha retroalimentación.

También se ha evaluado si la revisión y corrección entre pares o asistidos por un sistema de instrucción personalizado incrementa la realización de las tareas programadas en un curso (Crone-Todd, Eyre, Hutchens, Jones, \& Pear, 2007) y se ha encontrado que aquellos participantes que reciben la oportunidad de corregir sus errores, realizan más tareas programadas en el curso que aquellos que no. Por último, se han evaluado los efectos de restringir el contacto visual en la escritura y revisión de textos (Pacheco \& Ortega, 2011; Pacheco, Ortega, \& Carpio, 2011); o bien evaluar el efecto de las respuestas de los lectores a lo escrito sobre la revisión o modificación del documento (Pacheco, 


\section{Evaluación del modo lingüístico escribir con posibilidad de revisión y corrección del texto}

Ortega, \& Carpio, 2013) encontrado estos autores modificaciones en aspectos de forma pero no en contenido.

Se deriva de los estudios citados el papel relevante que la revisión y corrección tienen sobre la escritura de textos. Conforme se les pone en práctica, se señalan también como un indicador de dominio de la escritura. Es decir, que las personas que revisan y corrigen sus textos tienden a cometer menos errores en los textos, principalmente en aspectos de forma, pero también en el contenido, como muestran los resultados de estos estudios.

En vista también de que en los estudios de habilitación lingüística de la escritura de López et al. (2018) y López et al. (2017) se restringió la posibilidad de que los participantes revisaran y corrigieran su texto al tener que escribirlo de nueva cuenta en la postprueba, y visto que la revisión y corrección tienen un papel relevante en la escritura, se considera que al facilitar la opción de revisión (de hacer contacto de nueva cuenta con el texto escrito después de exponer a los participantes a los modos reactivos) y proporcionar así la posibilidad de corrección, facilitaría la habilitación lingüística de la escritura y observar sus efectos en los aspectos de forma y contenido en los textos a escribir. Es por ello que el propósito del presente estudio fue evaluar la exposición de estudiantes universitarios a los modos lingüísticos reactivos, sobre el modo activo escribir, con la opción de revisión y corrección de los textos escritos en la preprueba, al presentarlos de nueva cuenta en la postprueba.

\section{Método}

\section{Participantes}

Participaron de manera voluntaria 15 estudiantes (todas mujeres) del segundo semestre de la carrera de psicología de una institución pública de la ciudad de Hermosillo, Sonora. El promedio de edad de los participantes fue de 19.6 años, en un rango de 18 a 25 años.

\section{Aparatos y situación experimental}

El estudio se realizó con un programa creado bajo ambiente Microsoft Visual Basic Express 2010. Las sesiones experimentales se condujeron en el Laboratorio de Ciencia y Comportamiento Humano del Departamento de Psicología y Ciencias de la Comunicación de la Universidad de Sonora, en una sala aislada de ruidos y equipada con 20 computadoras de escritorio con CPU Titán de 500 GB y monitor, mouse y teclado LANIX,instaladas en cubículos individualizados para realizar de forma separada las actividades programadas durante la sesión. En los casos en los que los participantes requirieron escuchar, se utilizaron audífonos de la marca HP y Steren.

\section{Diseño experimental}

Se utilizó un diseño experimental de tres grupos con preprueba y postprueba, donde se evaluó la habilitación lingüística de la escritura a partir de cinco categorías que se describen en procedimiento, así como los errores deforma corregidos; esto a partir de la exposición a los modos reactivos escuchar, leer y observar, y la presentación del texto escrito en la preprueba para su revisión y posible corrección en la postprueba. El diseño estuvo conformado por tres fases: preprueba, exposición al modo reactivo y prueba de habilitación o postprueba (ver Tabla 1). Los 15 participantes fueron distribuidos en 3 grupos de 5 participantes cada uno, los cuales se denominaron para su identificación como Grupo Audio, Grupo Texto y Grupo Video. La asignación se hizo de forma alternada de acuerdo con el orden de llegada de los participantes a la sala experimental: el primer participante en llegar se asignó al Grupo Audio; el segundo participante al Grupo texto; el tercer participante al Grupo Video; los participantes subsiguientes se asignaron en el mismo orden. Ninguna de las actividades dentro de la sesión experimental tuvo tiempo límite y los participantes podían tomarse el tiempo que consideraran necesario para ejecutarlas.

Tabla 1. Diseño Experimental para la evaluación de la habilitación lingüística del modo activo escribir con corrección.

\begin{tabular}{|c|c|c|c|}
\hline Grupos $(n=5)$ & Preprueba & $\begin{array}{l}\text { Exposición modo } \\
\text { reactivo }\end{array}$ & Postprueba \\
\hline Grupo Audio & \multirow{3}{*}{$\begin{array}{r}\text { Escribir un texto sobre } \\
\text { el ciclo del agua }\end{array}$} & Escuchar & \multirow{3}{*}{$\begin{array}{l}\text { Presentación del texto escrito en la } \\
\text { preprueba para corrección o } \\
\text { reescritura }\end{array}$} \\
\hline Grupo Texto & & Leer & \\
\hline Grupo Video & & Observar & \\
\hline
\end{tabular}

Fuente: Elaboración propia

\section{Procedimiento}

Teniendo como base el procedimiento empleado por Tamayo et al. (2010) Tamayo y Martínez (2014), los participantes pasaron por la fase de preprueba, donde debían escribir un texto de extensión libre sobre el tema El ciclo del agua. Posteriormente, en la segunda fase o exposición al modo reactivo, fueron expuestos al modo reactivo correspondiente de la siguiente manera:

Los participantes del Grupo Texto debían leer un documento de 810 palabras que describía el ciclo del agua y los principales fenómenos asociados con él.
Los participantes del Grupo Audio debían escuchar una grabación de sonido de seis minutos de duración que describía el ciclo del agua y los principales fenómenos asociados con él, y que fue realizada a partir del texto impreso, es decir, fue exactamente el mismo contenido presentado de modo distinto.

Los participantes del Grupo Video debían observar un video de tres minutos de duración que representaba el ciclo del agua y los principales fenómenos asociados con él. El video no contenía audio ni subtítulos. Cabe aclarar que, aunque no se consideró este grupo como igualado o similar a los de la condición de Audio y Texto por carecer de las explicaciones pertinentes en forma textual o auditiva, el ciclo y los fenómenos se representaron en el mismo orden en que se describían en los otros dos grupos. Asimismo, se consideró que los participantes, 
al encontrarse cursando estudios profesionales, ya habían tenido contacto previamente en sus estudios preparatorios con la descripción del fenómeno.

Por último, todos los participantes fueron expuestos a la prueba de habilitación o postprueba, donde se les presentaba el texto escrito en la preprueba para que revisaran y corrigieran o reescribieran en caso de que así lo consideraran necesario.

La sesión experimental se condujo de la siguiente manera:

Después de tomar los datos generales del participante, éste era asignado a una computadora de escritorio en un cubículo aislado. Es importante mencionar que a cada participante se le entregó un formato para obtener su consentimiento informado, el cual describía el objetivo general del estudio, las sesiones a las que debía asistir de ser necesario, las actividades a realizar, el tiempo estimado que podrían llevar las tareas, así como los aspectos vinculados a la confidencialidad y el manejo responsable de la información. Por último, se explicaba que podía retirarse de la sesión o del estudio cuando así lo decidiera, sin ninguna consecuencia. Enseguida, se le indicaba que el mismo programa le proporcionaría las instrucciones, las cuales tenían que leer cuidadosamente para realizar la tarea. Se les preguntaba si tenían alguna duda o consulta antes de comenzar.

El programa iniciaba, daba la bienvenida, pedía los datos del participante y explicaba la finalidad de la actividad. Una vez hecho esto podía comenzar.

Las instrucciones (así como las categorías de medida) que se presentaron corresponden a las usadas por López et al. (2017) y únicamente se diferenciaron respecto la instrucción correspondiente a la fase 3 o Exposición al modo reactivo, que se modificó para indicar a los participantes la finalidad en dicha fase.

Instrucción 1 para preprueba a los tres grupos, Audio, Texto y Video: A continuación se te presenta una pantalla en blanco en la que tienes que escribir todo lo que sepas o recuerdes acerca del ciclo del agua o ciclo hidrológico como también le llaman. Puedes tomarte el tiempo que consideres necesario para hacerlo. Trata de escribir de la mejor manera posible, cuidando no cometer errores ortográficos. Una vez que hayas terminado y estés listo para continuar, presiona siguiente. Una vez que hayas presionado siguiente ya no podrás volver a hacer modificaciones en lo que escribiste.

Instrucción 2 para exposición al modo reactivo para el Grupo Audio: A continuación se te presenta un audio donde se describe el ciclo del agua, el cual te servirá de ayuda para que lo escribas nuevamente una vez que lo hayas escuchado. Puedes escucharlo las veces que lo consideres suficiente, sólo daleclick en reproducir. Una vez que hayas terminado y estés listo para continuar, presiona siguiente. Una vez que hayas presionado siguiente ya no podrás volver a reproducir el audio.

La instrucción 2 para los grupos Texto y Video fue similar, con la diferencia de que se les indicaba que se les presentaría un texto o un video, según la condición correspondiente.

Instrucción 3 para exposición al modo reactivo para el Grupo Audio: A continuación se te presenta el texto que escribiste hace un momento. Con base en lo escuchado realiza las correcciones que consideres necesarias para mejorar tu escrito sobre el ciclo del agua. Puedes tomarte el tiempo que consideres necesario para hacerlo.

De igual forma, la instrucción 3 para los grupos de Texto y Video fue similar, con la diferencia en la indicación respecto a la condición a la que acababan de ser expuestos.

\section{Categorías y criterios de medida}

Las categorías usadas para evaluar los productos escritos consideran tanto aspectos morfológicos como funcionales de los textos. Las categorías fueron: Mención de conceptos técnicos, Conceptos técnicos utilizados de manera pertinente y Frases referidas a relaciones. Para esta última, las relaciones que conformaron esta categoría fueron: relaciones condicionales, relaciones de uso o finalidad, relaciones causales, relaciones comparativas y relaciones de contraste Acuña et al, (2013; Mares, Rivas, \& Bazan, 2002). También se utilizó la categoría Frases formuladas, como una manera de identificar y comparar la extensión de la referencia escrita, pero con un sentido gramatical completo. Por último, se usó la categoría Instancias descriptivas contenidas dentro de las frases. La identificación y medición de esta última se hizo a partir de los sustantivos que componen las frases y que son independientes del sujeto y el verbo. Por ejemplo, en las siguientes frases que refieren al proceso de evaporación en el ciclo del agua: El sol calienta el agua y Los rayos del sol calientan el agua de océanos, ríos y lagos, se identifica que la segunda contiene más elementos descriptivos y por ello se considera más precisa acerca del referente del que se escribe Lopez (2017). De esta manera, las categorías para identificar aspectos de forma fueron Mención de conceptos Técnicos y Frases formuladas. Para identificar aspectos funcionales las categorías correspondieron a Conceptos técnicos utilizados de manera pertinente, Instancias descriptivas contenidas dentro de las frases y Frases referidas a relaciones. La calificación se otorgaba a partir de la identificación de las categorías. Se otorgaba un punto por categoría identificada en los textos revisados (ver Tabla 2). 
Evaluación del modo lingüístico escribir con posibilidad de revisión y corrección del texto

Tabla 2.Categorías de medida.

\begin{tabular}{|c|c|c|}
\hline Categorías & Descripción & Ejemplos \\
\hline Mención de conceptos técnicos (MC) & $\begin{array}{l}\text { Hace mención de términos técnicos } \\
\text { vinculados con el material. }\end{array}$ & Precipitación \\
\hline Frases formuladas (FF) & $\begin{array}{l}\text { Frases formuladas con un sentido } \\
\text { gramatical completo, identificadas } \\
\text { a partir del verbo. }\end{array}$ & Los rayos del sol calientan el agua. \\
\hline $\begin{array}{r}\text { Conceptos técnicos utilizados de } \\
\text { manera pertinente }(\mathrm{CP})\end{array}$ & $\begin{array}{l}\text { Utiliza los términos técnicos para } \\
\text { describir un evento/suceso o alguna } \\
\text { relación entre eventos. }\end{array}$ & $\begin{array}{l}\text { El agua en forma de vapor sube y se } \\
\text { condensa formando las nubes. }\end{array}$ \\
\hline $\begin{array}{r}\text { Instancias descriptivas contenidas } \\
\text { dentro de las frases (IDF) }\end{array}$ & $\begin{array}{l}\text { Número de sustantivos utilizados en } \\
\text { la frase independientes al sujeto de } \\
\text { la oración. }\end{array}$ & Los rayos del sol calientan el agua. \\
\hline
\end{tabular}

Las relaciones podían ser de tipo Condicionales: cuando, si, siempre que, a condición de que, supongamos, una vez que.

Causales: por (medio de), porque, puesto que, debido a/lo que, ya que, dado que, visto que, en efecto, en consecuencia, por condicional, causal, funcional (uso o consiguiente, por lo tanto, de ahí que. Frases referidas a relaciones (FR) finalidad), comparación y contraste. Éstas se identificaban a partir de los conectivos correspondientes a cada relación.
Funcionales (uso o finalidad): para, para que, a fin de que, con objeto de, con la intención de.

Comparación: como, al igual que, del mismo modo, de modo similar.

Contraste: pero, sin embargo, ahora bien, aunque, a diferencia, mientras que, no obstante, en cambio, sino (que).

Por último, se midieron el número de errores de forma y el número de correcciones hechas a los mismos. Por errores de forma se consideraron errores gramaticales de los siguientes tipos: palabras mal escritas, falta de acento, uso de una letra por otra (b por v, por ejemplo) conjugación incorrecta del verbo o falta de concordancia entre el sujeto y el predicado.

\section{Resultados}

La revisión de los textos elaborados por los participantes fue realizada por tres observadores de manera independiente con base en las categorías de medida definidas. Una vez revisados los escritos se compararon los puntajes otorgados en cada categoría por participante y los casos en que hubo desacuerdo fueron discutidos y resueltos por consenso.

En la Figura 1 se presentan los puntajes obtenidos por cada uno de los participantes del Grupo Audio en preprueba (barra blanca) y en postprueba (barra negra) en cada una de las categorías, también se presenta el promedio de los puntajes obtenidos por todos los participantes del grupo. Se puede observar un incremento en los puntajes obtenidos por todos los participantes al pasar de la preprueba a la postprueba, este efecto fue mucho más pronunciado en las categorías de Frases formuladas (FF) e Instancias descriptivas en frases (IDF). Adicionalmente a las categorías de FF e IDF, el participante 2 mostró un incremento en la categoría de Frases referidas a relaciones (FR), el participante 4 en Mención de conceptos (MC) y Frases referidas a relaciones (FR), mientras que el participante 5 mostró un incremento en Mención de conceptos (MC) y Conceptos pertinentes (CP). 


\section{Grupo Audio}

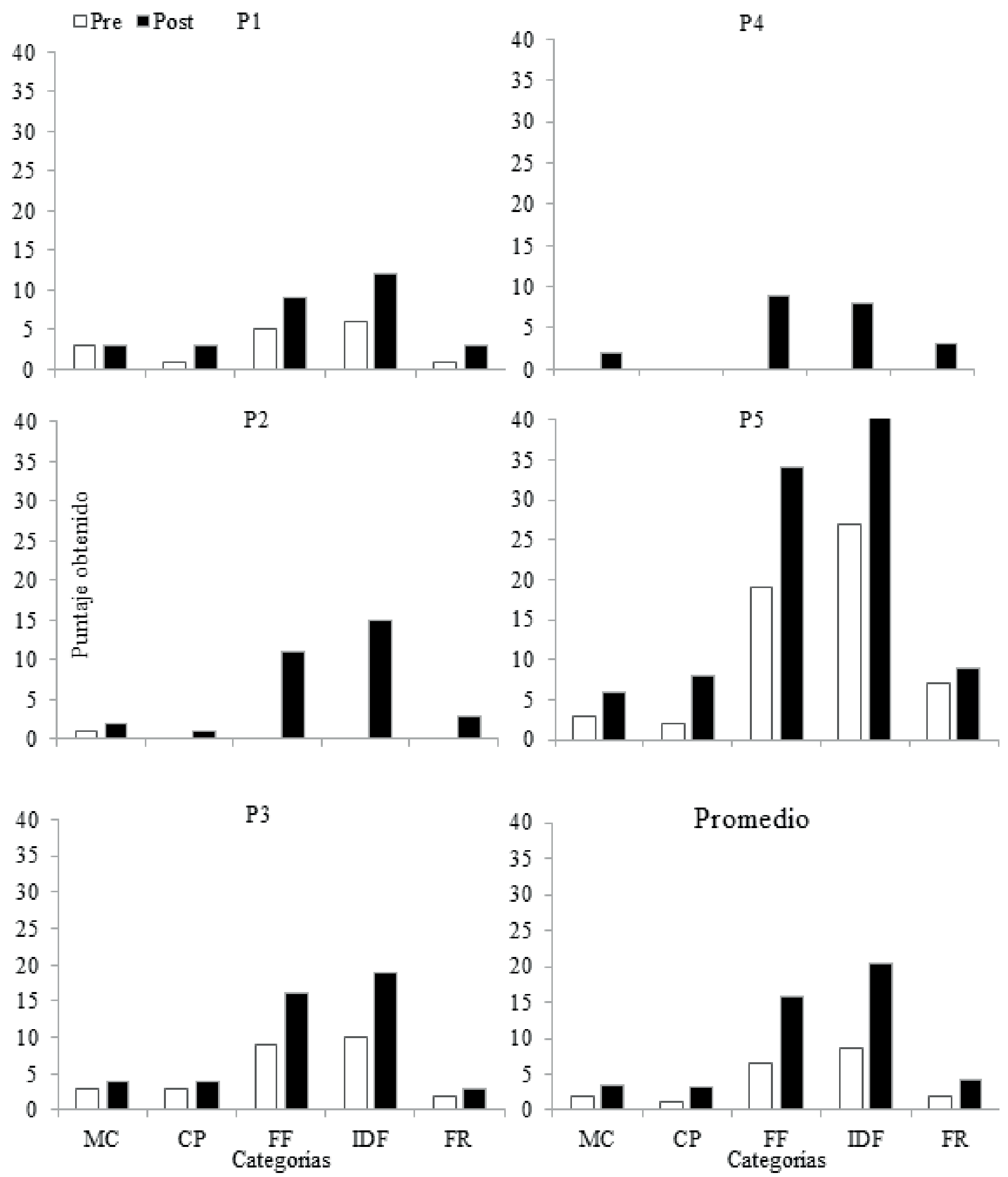

Figura 1. Puntaje obtenido en la preprueba y la postprueba para cada uno de los participantes y promedio del Grupo Audio en cada categoría: MC (mención de conceptos técnicos), FF (frases formuladas), CP (conceptos técnicos utilizados de manera pertinente), IDF (instancias descriptivas contenidas dentro de las frases), FR (frases referidas a relaciones -causales, condicionales, funcionales, comparativas y contraste).

En la Figura 2 se presentan los puntajes obtenidos por cada uno de los participantes del Grupo Texto en preprueba (barra blanca) y en postprueba (barra negra) en cada una de las categorías, también se presenta el promedio de los puntajes obtenidos por todos los participantes del grupo. Al igual que en los resultados del Grupo Audio, se puede observar un incremento en los puntajes obtenidos por todos los participantes al pasar de la preprueba a la postprueba, donde el efecto se observó más pronunciado en las categorías de Frases formuladas (FF) y en Instancias descriptivas contenidas dentro de las frases (IDF). Adicionalmente, los participantes 1, 4 y 5 mostraron un incremento en la categoría de Frases referidas a relaciones (FR). 


\section{Grupo Texto}
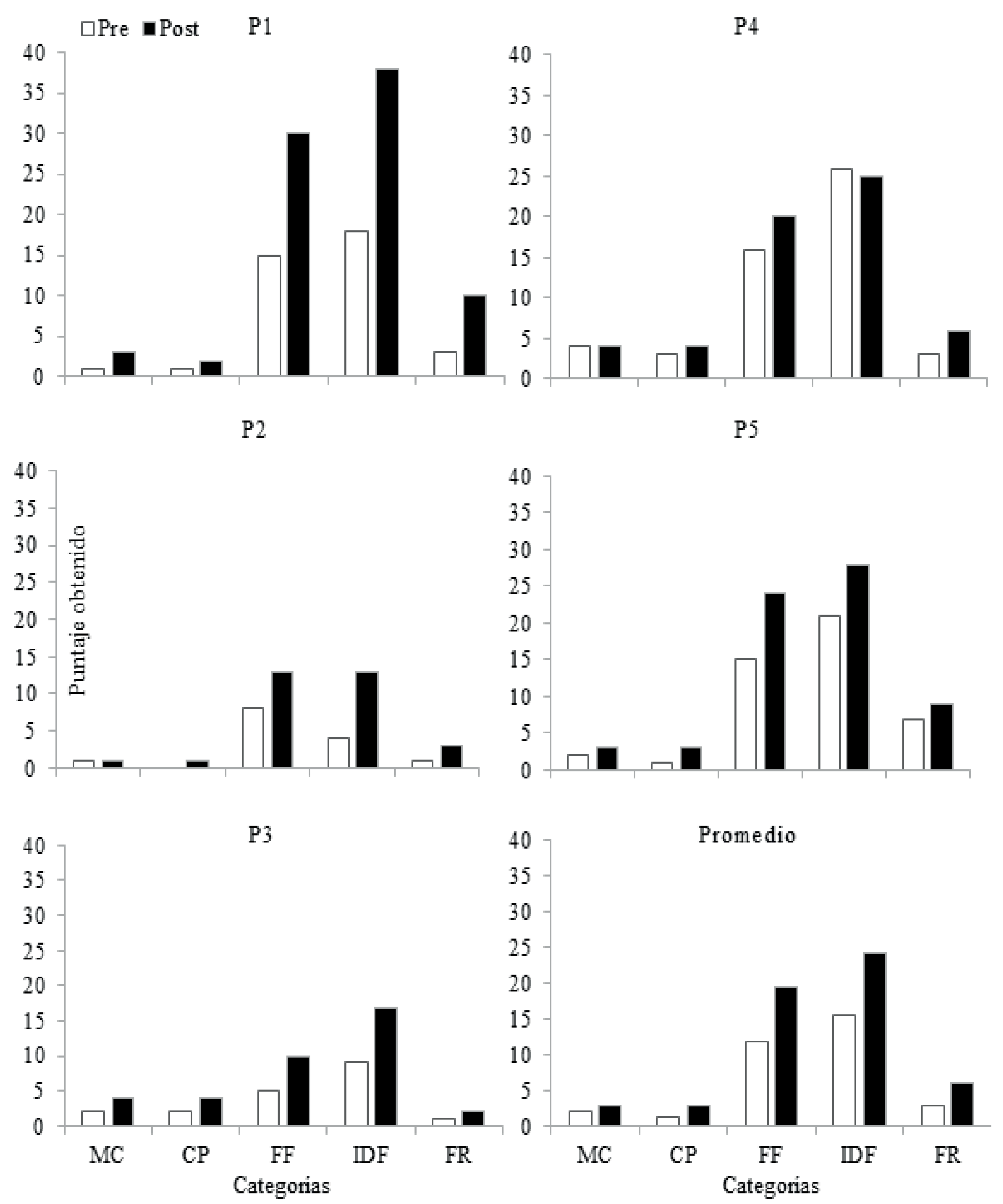

Figura 2. Puntaje obtenido en la preprueba y la postprueba para cada uno de los participantes y promedio del Grupo Texto en cada categoría: MC (mención de conceptos técnicos), FF (frases formuladas), CP (conceptos técnicos utilizados de manera pertinente), IDF (instancias descriptivas contenidas dentro de las frases), FR (frases referidas a relaciones -causales, condicionales, funcionales, comparativas y contraste).

En la Figura 3 se presentan los puntajes obtenidos por cada uno de los participantes del Grupo Video en preprueba (barra blanca) y en postprueba (barra negra) en cada una de las categorías, también se presenta el promedio de los puntajes obtenidos por todos los participantes del grupo. A diferencia de los resultados obtenidos en el Grupo Texto y en el Grupo Audio, en el caso del Grupo Video los incrementos en los puntajes obtenidos al pasar de la preprueba a la postprueba no fueron consistentes para los diferentes participantes en todas las categorías. Sin embargo, se mostró un incremento en todos los participantes en las categorías de Frases formuladas (FF), mientras que en Instancias descriptivas contenidas dentro de las frases (IDF) fue para tres de los cinco participantes, el 1, 2 y 5. 


\section{Grupo Video}

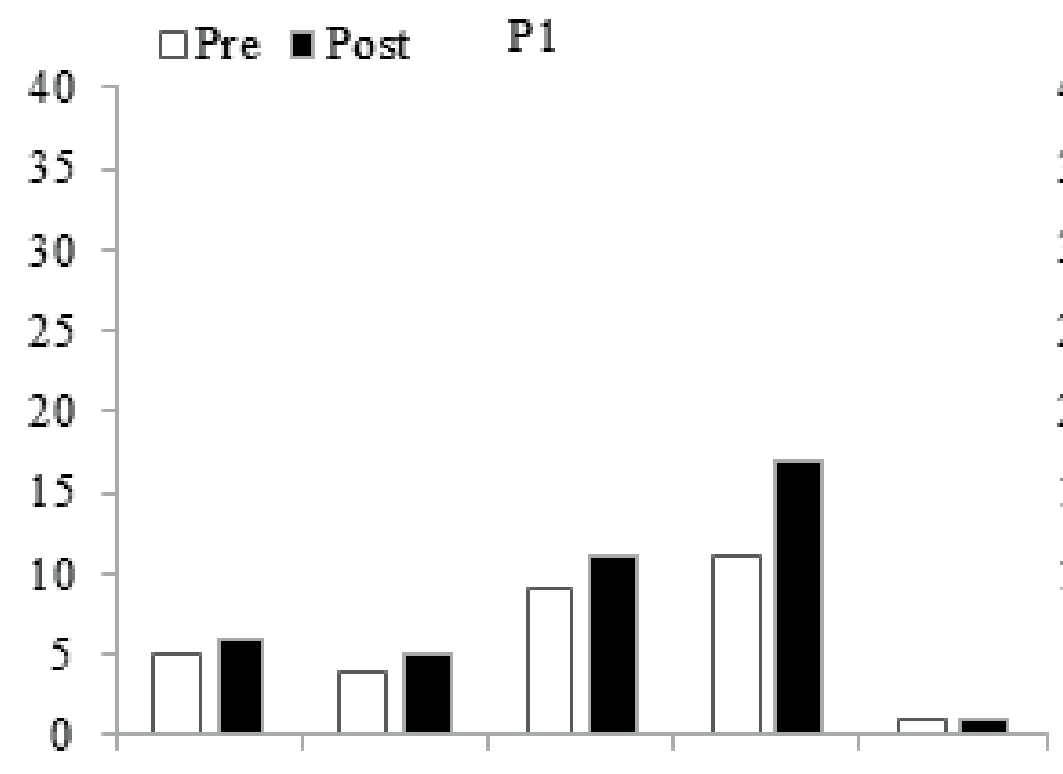

$\mathrm{P} 2$
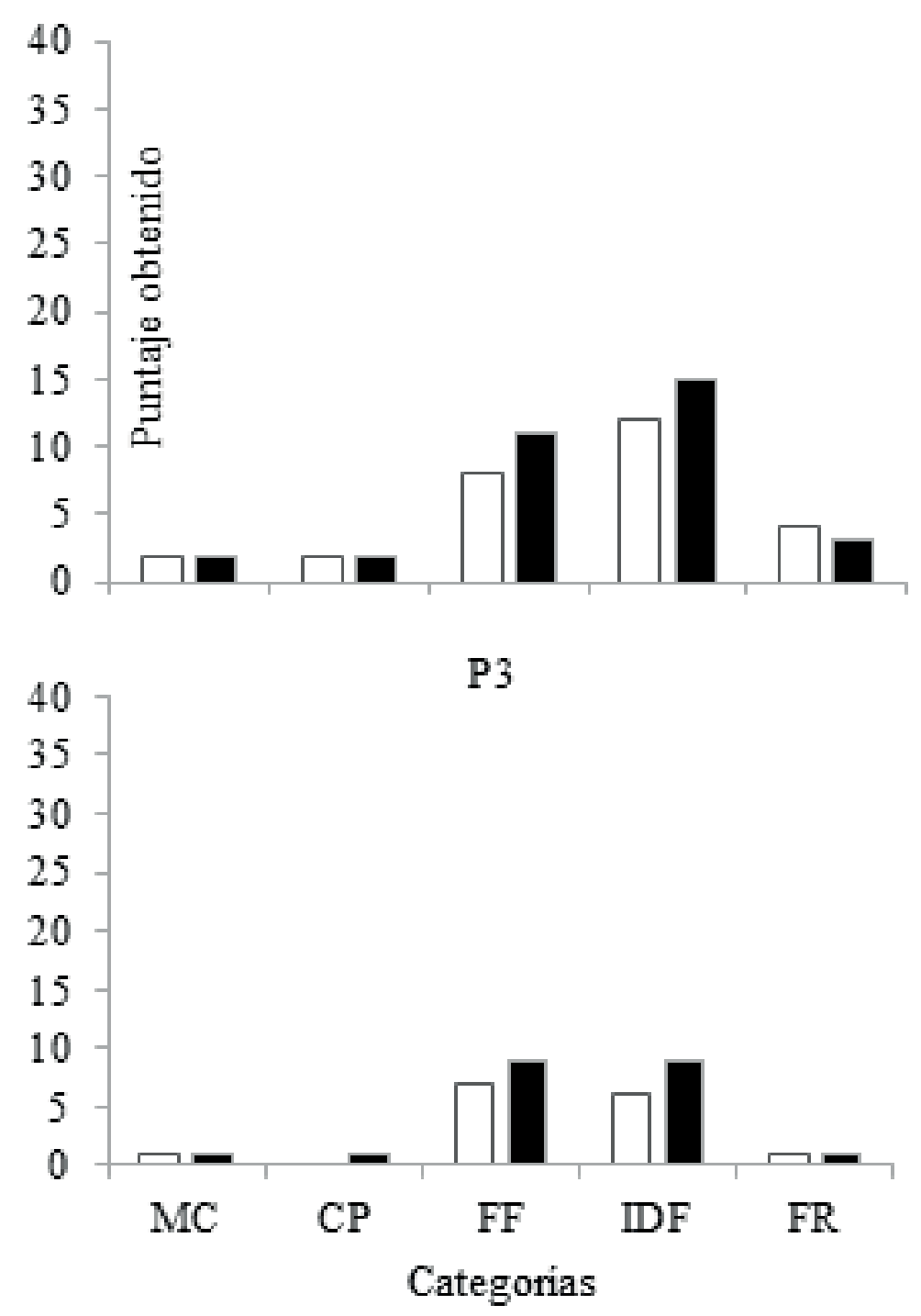

P4

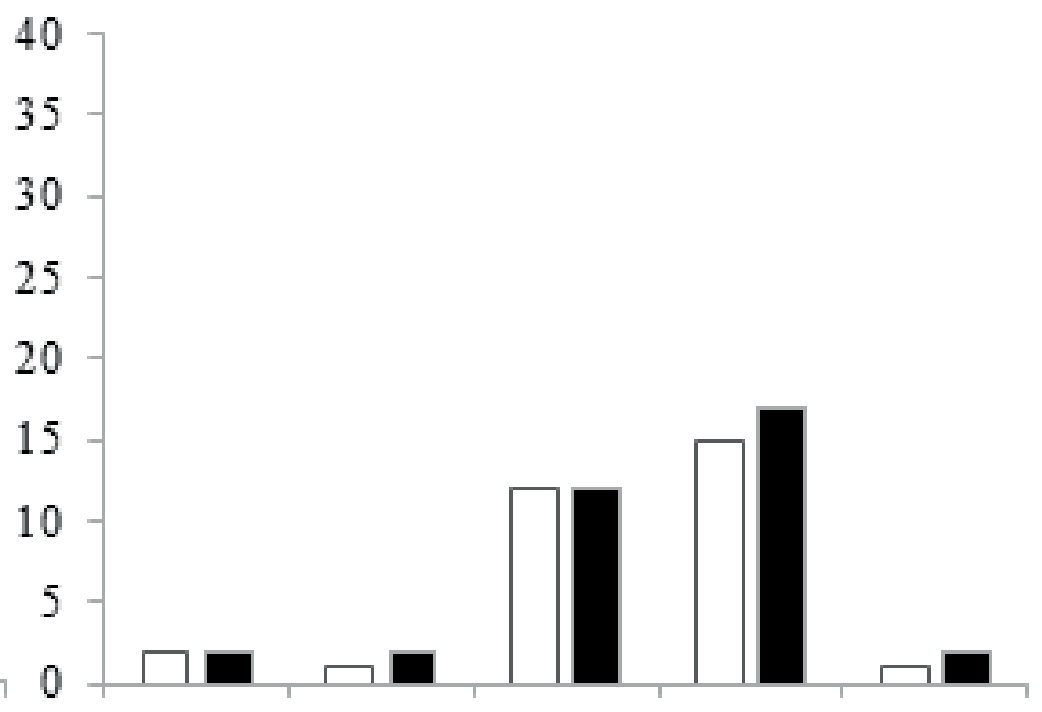

P5
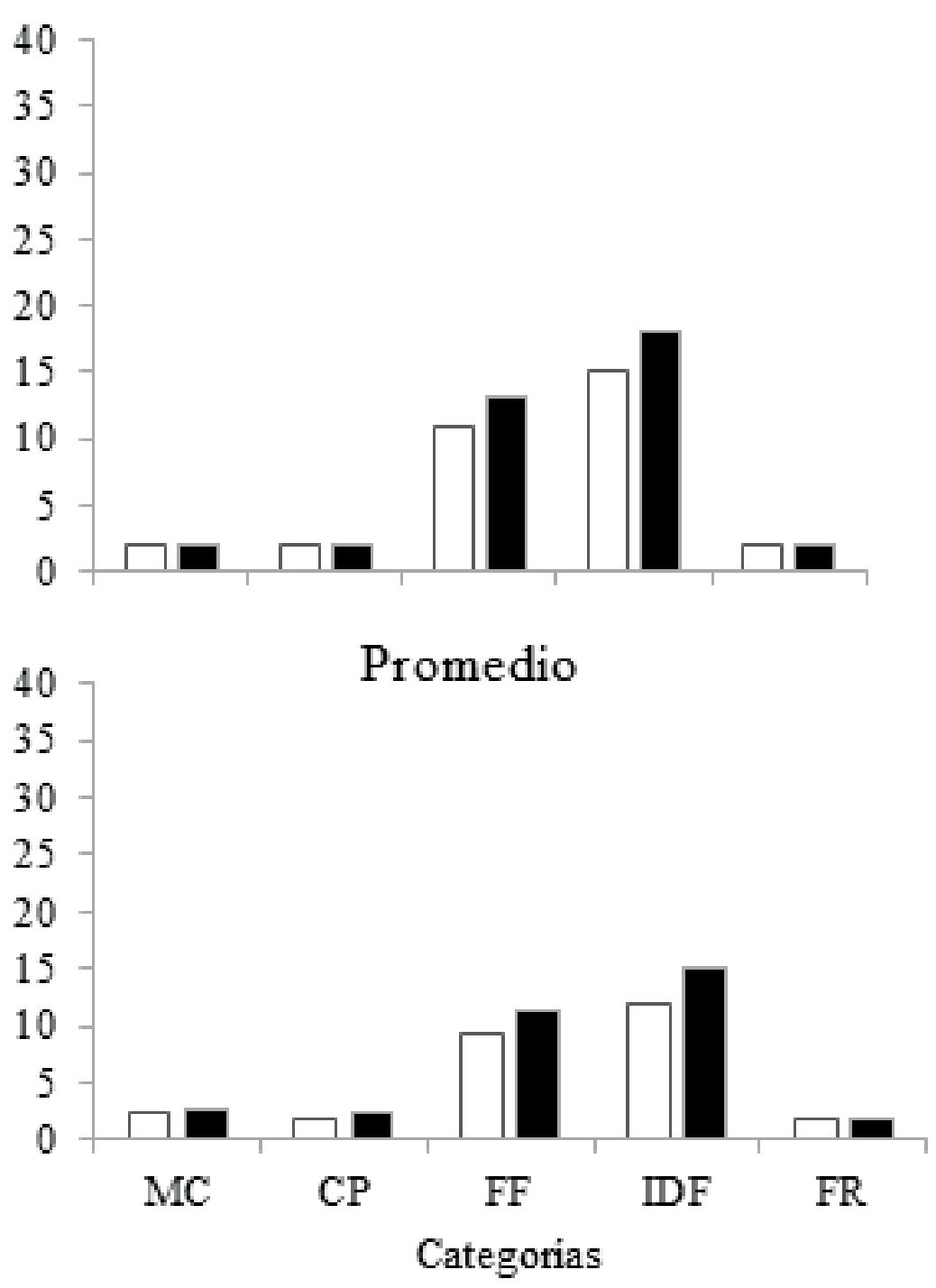

Figura 3. Puntaje obtenido en la preprueba y la postprueba para cada uno de los participantes y promedio del Grupo Video en cada categoría: MC (mención de

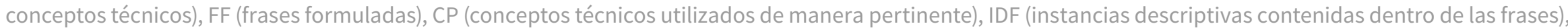
FR (frases referidas a relaciones -causales, condicionales, funcionales, comparativas y contraste).

En la Figura 4 se presenta el desempeño promedio por grupo, fase y categoría. En la ejecución de los tres grupos se muestran tendencias ascendentes pronunciadas en las categorías de Frases formuladas (FF) e Instancias descriptivas contenidas dentro de las frases (IDF), al pasar de la preprueba a la postprueba. Lo que indican estos resultados es un contacto más de tipo instancial con los referentes de los cuales se ve, lee o escucha, aun cuando se observó una tendencia ligeramente ascendente para el grupo de Texto y para el grupo de Audio en categorías como Frases referidas a relaciones (FR) o Conceptos pertinentes (CP). 


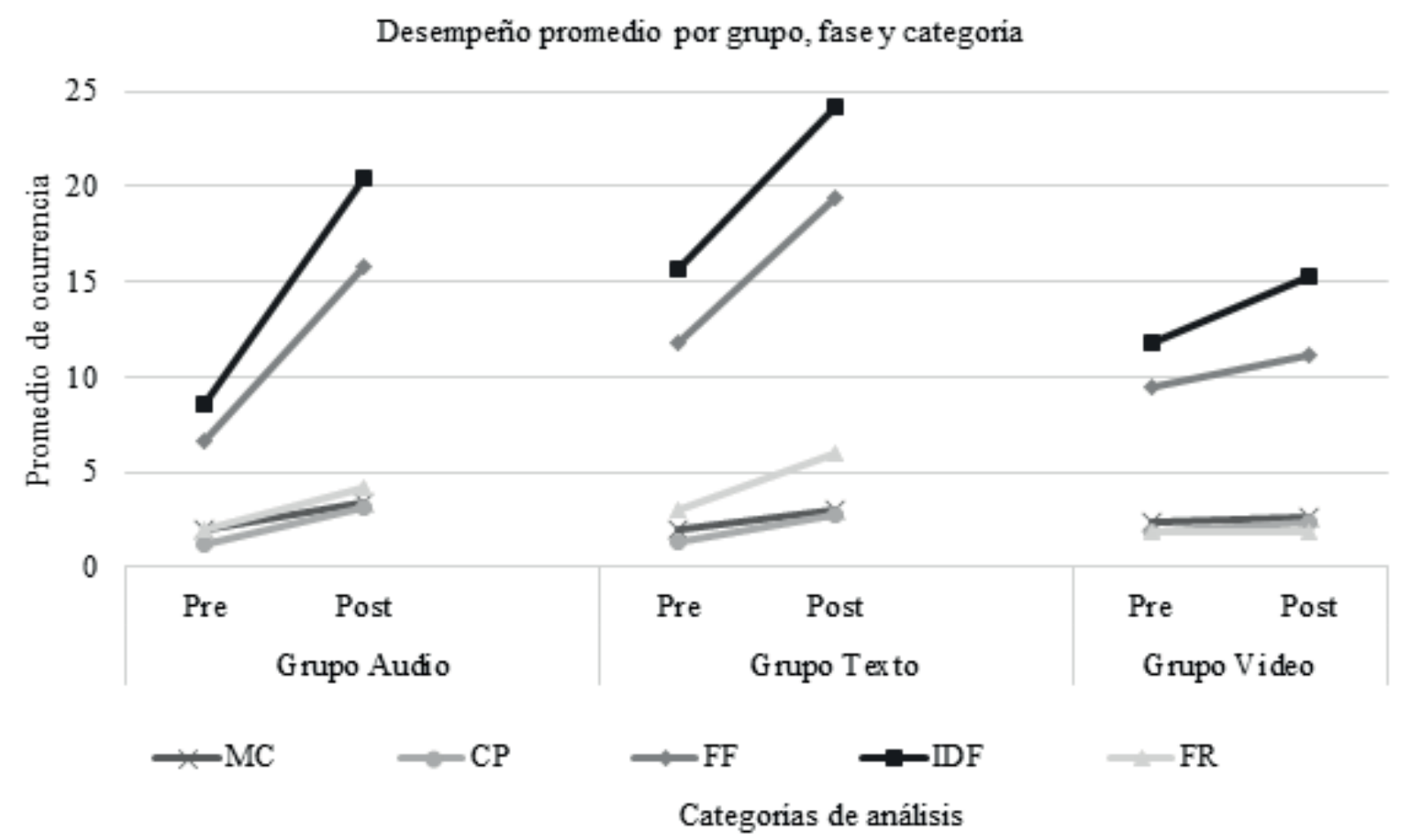

Figura 4. Desempeño promedio por grupo, fase y categoría: MC (mención de conceptos técnicos), FF (frases formuladas), CP (conceptos técnicos utilizados de manera pertinente), IDF (instancias descriptivas contenidas dentro de las frases), FR (frases referidas a relaciones -causales, condicionales, funcionales, comparativas y contraste).

En la Tabla 3 se muestran los errores de forma cometidos y corregidos por cada participante de los grupos Audio, Texto y Video, así como el total de errores por grupo. Se observa que ninguno de los participantes de los tres grupos corrigió alguno de los errores de forma cometidos en la preprueba. Los Grupo Audio y Texto cometieron adicionalmente más errores en la postprueba que en la preprueba, mientras que el Grupo Video cometió menos errores en la postprueba. Debe señalarse que los errores adicionales cometidos en la postprueba parecen estar en función del texto escrito de forma adicional en dicha condición (entre más texto añadido al documento escrito en la Tabla 3. Errores de forma cometidos y corregidos preprueba, se encontraron más errores adicionales), probablemente por ello tanto los participantes de los grupos Audio y Texto cometieron más errores de forma que los participantes del Grupo Video, donde la extensión de sus textos fue menor. De manera particular, se muestra que el participante 3 del Grupo Audio reescribió completamente el texto hecho en la preprueba, pero adicionalmente cometió nueve errores. El participante 2 del Grupo Audio no cometió errores de forma en la preprueba, sin embargo, en el texto escrito en la postprueba cometió tres errores, mismo caso para el participante 1 del Grupo Video, sólo que éste último tampoco cometió errores en la postprueba.

\begin{tabular}{|c|c|c|c|c|}
\hline Grupo & Participante & EPRE & ECR & EPOST \\
\hline \multirow{6}{*}{ Audio } & 1 & 4 & 0 & 3 \\
\hline & 2 & 3 & 0 & 8 \\
\hline & 3 & 5 & RET & 9 \\
\hline & 4 & 1 & 0 & 8 \\
\hline & 5 & 16 & 0 & 14 \\
\hline & Total & 29 & 0 & 42 \\
\hline \multirow{6}{*}{ Texto } & 1 & 8 & 0 & 12 \\
\hline & 2 & 0 & NA & 3 \\
\hline & 3 & 4 & 0 & 5 \\
\hline & 4 & 6 & 0 & 8 \\
\hline & 5 & 1 & 0 & 1 \\
\hline & Total & 19 & 0 & 29 \\
\hline \multirow{6}{*}{ Video } & 1 & 0 & NA & 0 \\
\hline & 2 & 8 & 0 & 3 \\
\hline & 3 & 4 & 0 & 11 \\
\hline & 4 & 6 & 0 & 1 \\
\hline & 5 & 2 & 0 & 0 \\
\hline & Total & 20 & 0 & 15 \\
\hline
\end{tabular}

Nota:. Errores de forma por participante y por grupo cometidos en la preprueba, corregidos en la postprueba y errores de forma cometidos adicionalmente en la postprueba. EPRE (Errores cometidos en la preprueba), ECR (Errores corregidos en la postprueba), EPOST (Errores cometidos en la postprueba), RET (Reescritura 
Adicionalmente al tratamiento de los datos presentados, se realizó su análisis estadístico mediante la prueba Wilcoxon y el cálculo del tamaño del efecto. Wilcoxon es una prueba no paramétrica que considera dos grupos de mediciones efectuadas a un solo conjunto de individuos, con la condición de que haya una variable independiente de tipo cualitativo nominal con dos modalidades y una variable dependiente cuantitativa continua con distribución no semejante a la curva normal, o de tipo cuantitativo discreto, y que es aplicable en muestras pequeñas (Valenzuela, 2006). En este caso se aplicó al contar con datos continuos pero que se comportan como discretos (son pocos datos y se muestran escalonados). En el caso de esta prueba se recomienda que el nivel de significancia no deba rebasar el 0.05 .

Respecto al tamaño del efecto, éste cuantifica la magnitud de la diferencia entre dos medias y representa el grado en que la hipótesis nula es falsa; cuando el tamaño del efecto es grande, la potencia estadística aumenta. El tamaño del efecto refiere al resultado de un tratamiento experimental que se está dispuesto a aceptar como Tabla 4. Prueba Wilcoxon clínicamente relevante e indica cuánto de la variable dependiente se puede explicar, predecir o controlar por la variable dependiente (Cardenas \& Arancibia, 2014).

En el caso del tamaño del efecto, para las pruebas t, se considera .20 pequeño, .50 mediano y .80 grande. En este caso se determinó el tamaño del efecto de las categorías que obtuvieron resultados significativos en la prueba de Wilcoxon. La prueba Wilcoxon se realizó con el programa estadístico SPSS v20 y el tamaño del efecto se calculó en programa estadístico G*Power v3.

En la Tabla 4 se presentan los resultados de la prueba Wilcoxon. En general, para el conjunto de categorías resaltadas en negro, puede considerarse que existe una diferencia antes y después de exponerlos al modo lingüístico reactivo correspondiente. Tres categorías de los grupos Audio y Texto son significativas. En cuanto al grupo Video, únicamente una categoría (Instancias descriptivas en frases) resultó significativa.

\begin{tabular}{|cccccc|}
\hline Grupo & MC & CP & FF & IDF & FR \\
\hline Audio & 0.066 & 0.066 & 0.043 & 0.043 & 0.041 \\
\hline Texto & 0.102 & 0.038 & 0.042 & 0.08 & 0.042 \\
\hline Video & 0.317 & 0.083 & 0.059 & 0.039 & 1 \\
\hline
\end{tabular}

Nota: Resultados de la prueba Wilcoxon realizada a los grupos Audio, Texto y Video. Categorías: Mención de conceptos (MC), Conceptos pertinentes (CP), Frases formuladas (FF), Instancias descriptivas en frases (IDF) y Frases referidas a relaciones (FR). Nivel de significancia aceptable hasta el 0.05.

En la Tabla 5 se presenta el tamaño del efecto obtenido únicamente de las categorías que obtuvieron puntaje significativo tras realizar la prueba Wilcoxon. Es decir, en aquellos grupos con categorías en blanco no se determinó el tamaño del efecto. Con base en este resultado se puede indicar que el tamaño del efecto es grande y la diferencia entre medias es verdadera en todas aquellas categorías consideradas.

Tabla 5.Tamaño del efecto

\begin{tabular}{|c|c|c|c|c|c|}
\hline Grupo & $M C$ & $\mathrm{CP}$ & FF & IDF & FR \\
\hline Audio & & & 2.22 & 1.85 & 2.41 \\
\hline Texto & & 2.46 & 1.65 & & 1.27 \\
\hline Video & & & & 2.18 & \\
\hline
\end{tabular}

Nota: Tamaño del efecto en aquellas categorías que obtuvieron un resultado significativo tras realizar la prueba Wilcoxon. Categorías: Mención de conceptos (MC), Conceptos pertinentes (CP), Frases formuladas (FF), Instancias descriptivas en frases (IDF) y Frases referidas a relaciones (FR).

\section{Discusión}

El propósito de este estudio fue evaluar la exposición de estudiantes universitarios a los modos lingüísticos reactivos, sobre el modo activo escribir, con la opción de revisión y corrección de los textos escritos en la preprueba, al presentarlos de nueva cuenta en la postprueba. En el estudio de López et al. (2017), Una vez que fueron expuestos a los modos reactivos, no tuvieron contacto con el texto que habían escrito en la preprueba, sino que tuvieron que escribir uno nuevo sobre el mismo tema, privándolos así de una de las ventajas que otorga la escritura, que es poder revisar los textos escritos una vez transcurrido un tiempo variable, generalmente con el fin de corregirlos o mejorarlos. Comoya se mencionó, el ejercicio de la revisión y corrección puede tener un efecto positivo (al intentar la corrección de lo escrito se busca adecuarlos a los objetivos que persigue el texto) en la escritura, tomándose además como un indicador de lo que suelen hacer escritores expertos.

Los resultados mostraron un incremento en una o dos categorías adicionales a las reportadas por López et al. (2017). Estos autores reportaron aumentos consistentes en Frases formuladas (FF) e Instancias descriptivas en frases (IDF) en dos de sus tres grupos evaluados (Audio y Texto).En el presente caso, en el Grupo Audio los participantes mostraron un efecto adicional en otras dos categorías, Frases referidas a relaciones (FR) y Conceptos pertinentes (CP), mientras que en el Grupo Texto se mostró en una categoría adicional, en Frases referidas a relaciones (FR).Asimismo, el aumento en las categorías de FF e IDF se obtuvo en los tres grupos (Audio, Texto y Video), no sólo en dos como en el estudio mencionado.

Esto puede discutirse en términos de que ahora los participantes, además de aumentar la referencia sobre el ciclo del agua y las descripciones que incluyen sobre los fenómenos que lo componen, establecen relaciones intrafenómeno o bien entre fenómenos, de ahí el aumento en la categoría de FR en los grupos Audio y Texto. Por ejemplo, el efecto de los rayos del sol sobre el agua para la evaporación, o bien la relación entre la evaporación y la condensación o entre la escorrentía y los mantos acuíferos, por mencionar algunos recurrentes en los textos revisados. Asimismo, aunque la categoría Mención de conceptos (MC) es baja en comparación con FR y CP, sí se muestra que con la mención de los conceptos se incrementa la delimitación pertinente de los mismos. 


\section{Evaluación del modo lingüístico escribir con posibilidad de revisión y corrección del texto}

Lo anterior da pie para señalar que la opción de hacer contacto de nueva cuenta con el texto escrito, de revisarlo y hasta corregirlo, después de haberse expuesto a los participantes a los modos reactivos en cada una de las condiciones asignadas, influyó en los resultados encontrados en este experimento, si bien no se mostró para el total de categorías como era lo esperado. Esto resulta relevante si se analizan los resultados del Grupo Video, donde no se observó un decremento en la postprueba, como sí se observó en el estudio de López et al. (2017). Además de que el grupo no mostró un decremento en los resultados, se observó un incremento en las categorías de Frases formuladas (FF) e Instancias descriptivas en frases (IDF).

Respecto a la literatura que señala que la revisión y corrección sólo tiene efectos en la forma del texto y no en el contenido (Storch, 2007; Truscott \& Hsu, 2008) y la literatura que indica que también el efecto se da en el contenido (Min, 2016) (Rahimi, 2013), los resultados obtenidos por parte de los tres gruposmostraron mejoras en forma. Aquí debe aclararse que esta mejora es sólo un aumento en extensión, pero no en precisión, es decir, se formularon más frases, pero no se corrigió su ortografía, como dan cuenta los errores cometidos en la postprueba. Respecto a contenido, no es posible concluir a partir de esta información que el efecto que se muestra es significativo, aunque la categoría de Frases referidas a relaciones (FR) muestre un incremento (por lo demás, por debajo de FF e IDF). Esto, aparte de que los participantes aumenten la extensión y descripción de su referencia, aunque ahora también hagan relaciones respecto a los fenómenos que mencionan y describen.

Debe mencionarse, respecto a la posibilidad de revisión y corrección, que ninguno de los participantes corrigió alguno de los errores de forma. Aumentaron la extensión de su referencia (de su escrito) pero no corrigieron. En todo caso, en el texto adicional escrito en la postprueba cometieron aún más errores que en la preprueba. Es decir, no parece ser condición suficiente presentar de nueva cuenta el texto escrito para que la revisión (entrar en contacto otra vez con un texto escrito previamente) dé pie a la corrección, incluso cabe discutir hasta qué punto la mera disposición del material para que se haga contacto de nueva cuenta con él se considera revisión (en el sentido de revisar o remirar algún objeto o evento conocido para descubrirle propiedades antes no advertidas), pues este no parece ser el caso.

El añadido de información al texto escrito en la preprueba lleva a la consideración de que la exposición al modo reactivo (principalmente en la condición escuchar y leer) facilitó el agregado de escritura para completar la idea del texto que escribieron previamente, sin embargo, no se tomaron el tiempo para corregir lo ya escrito ni lo escrito adicionalmente. No hubo corrección, por lo tanto, puede decirse que no hubo reescritura del texto (si bien el participante 3 del Grupo Audio reescribió el texto, lo hizo a partir de borrar todo lo escrito previamente y en el texto nuevo cometió más errores que en el anterior).

Es pertinente mencionar, respecto a los hallazgos en los grupos de Audio y Texto, que no se muestra un efecto en los resultados que permita afirmar que uno de los modos lingüísticos (escuchar - leer) tiene primacía sobre el otro. Por último, en el sentido de seguir explorando la revisión y corrección en la escritura, y en vista de los resultados obtenidos, se hace necesario explorar variables relacionadas que puedan promover la revisión y corrección de la escritura. Algunas variables podrían ser el trabajo en pares o diadas, la supervisión de un experto, la respuesta del lector al texto del escritor, o bien el señalamiento o resaltado de errores durante la revisión. Preparaciones de este tipo debieran de incluirse como parte de las secuencias didácticas regulares en la educación básica para promover la extensión funcional de la escritura en los niños, modificando así inercias de copiary pegary permitiendo mayores niveles de parafraseo, mayor cuidado en la presentación de su escrito y dándole a la escritura el sentido comunicativo que debiera tener.

\section{Conclusiones}

Al ser el objetivo de este estudio evaluar el modo activo escribir en su relación con los tres modos reactivos: escuchar, leer y observar, no se implementaron otras combinaciones para evaluar la relación entre los modos lingüísticos reactivos y activos que permitieran medir posibles diferencias en las ejecuciones al ser expuestos al material del ciclo del agua. No obstante, de acuerdo a la complementariedad de los modos lingüísticos (Fuentes \& Ribes, 2001) al agruparse en pares reactivos (que para escribir sea necesario leer, por ejemplo, o que para hablar sea necesario escuchar) y al nivel de dificultad de la ejecución de acuerdo a sus características morfológicas y a las relaciones de igualdad por semejanza o diferencia en una situación de práctica (Camacho \& Gomez, 2007) se esperaba que el par leer-escribir tuviera desempeños con mayor porcentaje de aciertos sobre los pares escuchar-escribir y observar-escribir, lo cual no sucedió de esa forma.

En todo caso, las ejecuciones similares observadas entre los grupos de Audio y Texto indicarían que si no se encuentran diferencias, esto se explique por las características del modo escuchar, del que su medio de ocurrencia es auditivo y, de acuerdo a sus características del par hablar-escuchar se tiene la posibilidad de reproducir el estímulo en circunstancias de tiempo y espacios distintos y por ello correlacionarse con los otros modos (escribir, por ejemplo) (Camacho \& Gomez, 2007). Se considera además que estos modos (habla-escuchar) permiten o facilitan el contacto con objetos de estímulo que son lingüísticos.

Conbaseen esto, es que probablementeal momento deescribir, la facilitación de este modo no mostró diferencias en la postprueba, aunque previamente los participantes hubiesen sido expuestos a escuchar o a leer sobre el ciclo del agua. Se puede añadir además que las restricciones de cada uno de los modos (tener presente el estímulo a la hora de leer y su relativa permanencia y lo efímero del audio a la hora de escuchar) se emparejaron al momento de exponer a los participantes a la postprueba, pues no escribieron con los materiales presentes. Asimismo, si bien la exposición del ciclo del agua ocurrió mediante modos reactivos distintos, lo expuesto trató exactamente lo mismo, pues del texto desarrollado con el tema del ciclo del agua, se hizo la grabación para exponerlo por audio.

Debe señalarse que la presentación del modo observar obedeció a dos razones. La primera es que el propósito general del estudio fue evaluar cómo se escribe después de hacer contactos con los referentes, y en este caso se trató de evaluar cada modo reactivo por sí mismo, es decir, sin combinación con alguno otro. En suma, se trató de evaluar cómo se escribe después de escuchar algo, después de leer algo y después de observar algo, en un interés de conocer el impacto de los contactos directos con los estímulos (los materiales referentes) al momento de escribir. La segunda razón fue que se sopesó que al contar con una muestra de estudiantes universitarios (y que por lo tanto han tenido previamente contacto con diferentes materias de conocimiento) éstos ya habrían hecho contacto previamente con el tema del ciclo del agua, es decir, que no se trataba de hacer contacto con un tema completamente novedoso, sino más bien con un tema restringido en su forma de descripción y explicación. En este sentido, 
se supuso, aunque con los resultados ya presentados, que la sola observación habilitaría la escritura.En este caso, las restricciones mismas del modo observar al implementarse en este experimento sin contar con una combinación con otros modos (como leer o escuchar) y las características del tema que debía desarrollarse, que era netamente lingüístico, en el sentido de que la representación del ciclo del agua no es algo que pueda observarse directamente, es decir por exposición directa a cada uno de los fenómenos que lo componen, sino que se trata de un desarrollo conceptual que explica varios eventos relacionados, influyó en las ejecuciones del Grupo Video. Para finalizar, esto último no quiere decir que el modo observar tenga que ser presentado siempre en combinación con otros modos para obtener desempeños adecuados, sino que la forma lingüística en la que se presenta la tarea y el entrenamiento en situaciones similares también influyen en las ejecuciones.

\section{Referencias}

Acuña, K., Irigoyen, J.J. \& Jiménez, M. (2013). La comprensión de contenidos científicos en estudiantes universitarios. Recuperado dehttps://www.uv.mx/rmipe/files/2014/05/La-compresnion-decontenidos-cientificos-en-estudiantes-universitarios.pdfISBN: 978-60796359-0-9

Beaugrande, R. (1987). Writing and meaning: Contexts of research. En A. Matsuhashi (ed), Writing in real time: Modelling production processes, 1-33. Norwood, NJ: Ablex. ISBN: -13:978-0-387-96703-5

Bereiter, C. \& Scardamalia, M. (1987). The psychology of written composition. Hillsdale, NJ: Lawrence Erlbaum Associates. ISBN 0-85859-647-5

Camacho, J. \& Gómez, D. (2007). Variación de los modos de lenguaje en la adquisición y transferencia de conocimiento. En J.J. Irigoyen, M. Jiménez \& K. Acuña. Enseñanza, Aprendizaje y Evaluación. Una aproximación a la Pedagogía de las Ciencias (pp. 105-135). Recuperado de https://www.researchgate.net/publication/299612462 Ensenanza Aprendizaje y Evaluacion Una aproximacion a la Pedagogia de las Ciencias\#pf69 ISBN: 970-689-344-X

Camacho, J., Irigoyen, J.J., Gómez, D., Jiménez, M. \& Acuña, K. (2007). Adquisición y transferencia de modos lingüísticos en tareas de discriminación condicional sin retroalimentación reactiva. Enseñanza e Investigación en Psicología, 12 (1), 79-91. Recuperado de http://www.redalyc.org/pdf/292/29212106.pdfISSN: 0185-1594

Cárdenas, M. \& Arancibia, H. (2014). Potencia estadística y cálculo del tamaño del efecto en $\mathrm{G}$ * Power: complementos a las pruebas de significación estadística y su aplicación en psicología. Salud y Sociedad, 5, 210-224. Recuperado de http://dx.doi.org/10.22199/ S07187475.2014.0002.00006 DOI: 10.22199/S07187475.2014.0002.00006

Cassany. D. (2010). Describir el escribir. Cómo se aprende a escribir. Barcelona: Paidós.Isbn: 8449326494

Chávez, F. (2013). Redacción avanzada. Un enfoque lingüístico. México: Pearson. ISBN: 9786073238175

Crone-Todd, D., Eyre, H., Hutchens, S., Jones, J. \& Pear, J. (2007). The effect of changing grading criteria on student writing. The Behavior Analyst Today, 8, 35-42.Recuperado de https://files.eric.ed.gov/fulltext/ EJ800968.pdfISSN: 1539-4352 (Electronic)

Fuentes, M.T. \& Ribes, E. (2001). Un análisis funcional de la comprensión lectora como interacción conductual. Revista Latina de Pensamiento y Lenguaje, 9, 181-212.ISSN 0123-

Gómez, D. (2005). Transferencia entre modos del lenguaje y niveles de interacción: observar, escuchar, hablar, leer y escribir. Tesis Doctoral Inédita. Centro de Estudios e Investigaciones en Comportamiento. Universidad de Guadalajara.DOI:10.5460/jbhsi.v6.1.47599 ISSN: 2007$\underline{0780}$
Gómez, A. D. \& Ribes, E. (2008). Adquisición y transferencia de una discriminación condicional de primer orden en distintos modos del lenguaje. Acta Comportamentalia, 16, 183-209.doi:10.5460/jbhsi. v6.1.47606

Hayes, J. R. \& Flower, L. S. (1980). Identifying the organization of writing processes. En L. Gregg \& E. Steinberg (eds.), Cognitive process in writing: An interdisciplinary approach, 3-30. Hillsdale, NJ: Lawrence Erlbaum Associates.DOI: 10.1093/acprof:0so/9780199827282.003.0001

Hyland, F. (2003). Focusing on form: student engagement with teacher feedback. System, 31, 217-230. doi: 10.1016/S0346-251X(03)00021-6

Kiuhara, S., Graham, S., \& Hawken, L. (2009). Teaching writing to high school students: a national survey. Journal of Educational Psychology, 101, 136-160. doi: 10.1037/a0013097ISSN:0022-0663

López, A., Flores, C. \& Irigoyen, J.J. (2018). Habilitación lingüística de la escritura y explicitación de instrucciones. Enseñanza e Investigación en Psicología, 23, 341-350. Recuperadode https://www.academia. edu/37209823/López A. Flores C. Irigoyen J.J. 2018 . Habilitación linguística_de_la_escritura_y_explicitación_de_instrucciones. Enseñanza_e_Investigación_en_Psicología_23_3_341-350

López, A., Flores, C. \&Torres, C. (2017). Análisis de la habilitación lingüística de la escritura. En J.J. Irigoyen, K. Acuña \& M. Jiménez. Aportes conceptuales y derivaciones tecnológicas en Psicología y Educación(pp. 259-280). Recuperado de http://www.qartuppi. com/2017/APORTES.pdfISBN 978-607-97326-8-4

Mares, G., Rivas, O.\& Bazán, A. (2002). Configuración en el modo escrito de competencias desarrolladas en forma oral como efecto del nivel funcional de ejercicio. Revista Mexicana de Análisis de la Conducta, 28, 173-202.Recuperado de http://dx.doi.org/10.5514/rmac.v28. i2.26328ISSN: 0185-4534

Min, H. (2016). Effect of teacher modeling and feedback on EFL students' peer review skills in peer review training. Journal of Second Language Writing, 31, 43-57.Recuperado de https://doi.org/10.1016/j. jslw.2016.01.004 DOI: 10.1016/j.jslw.2016.01.004

Pacheco, V. \& Ortega, M. (2011). El papel del contacto visual en la elaboración de textos con niños de educación básica. En J.J. Irigoyen, K. Acuña \& M. Jiménez (Coords.), Evaluación de desempeños académicos (pp. 27-42). Recuperado de http://WwW. interactum.com.mx/docs/DESEMPENOS.pdf ISBN 978-607-7782-96-4

Pacheco, V., Ortega, M.\& Carpio, C. (2011). Composición escrita en universitarios: papel del contacto visual en la revisión de textos. Suma Psicológica, 18, 29-40.Recuperado de: http://www.redalyc.org/ pdf/1342/134222985003.pdfISSN: 0121-4381

Pacheco, V., Ortega, M.\& Carpio, C. (2013). Efectos de la respuesta del lector y del uso de ejemplos sobre la composición escrita. Revista Colombiana de Psicología, 22, 13-34. Recuperado de https://revistas. unal.edu.co/index.php/psicologia/article/view/20989/43283ISSN: electrónico 2344-8644. ISSN: impreso 0121-5469.

Pulido, L. \& Tamayo, J. (2015). Aprendizaje comprensivo: evaluación de la habilitación lingüística en modos no complementarios. Revista Mexicana de Investigación en Psicología, 7, 52-66. Recuperado de https://www.researchgate.net/publication/281294216 Aprendizaje comprensivo evaluacion de la habilitacion linguistica en modos no complementarios ISSN-impresa: 2007-0926; ISSN-digital: 20073240

Quiroga, L., Peña, T. \& Padilla, M.A. (2013). Efectos del tipo de entrenamiento y del modo lingüístico sobre el ajuste a contingencias convencionales. Acta comportamentalia, 21, 66-82. Recuperado de http://www. redalyc.org/articulo.oa?id=274526149002ISSN: 0188-8145

Rahimi, M. (2013). Is training student reviewers worth its while? A study of how training influences the quality of students' feedback and writing. Language Teaching Research.17,67-89. Recuperado de http://www.redalyc.org/ articulo.oa?id=274526149002 DOI: $10.1177 / 1362168812459151$ 


\section{Evaluación del modo lingüístico escribir con posibilidad de revisión y corrección del texto}

Storch, N. (2007). Investigating the merits of pair work on a text editing task in ESL classes. LanguageTeachingResearch, 11, 143-159. Recuperado de http://dx.doi.org/10.1177/1362168807074600 DOI: $10.1177 / 1362168807074600$

Tamayo, J. \& Martínez, D. (2014). Efecto de habilitación en un procedimiento de igualación de la muestra de segundo orden. Revista Mexicana de Investigación en Psicología, 6, 97-108. Recuperado de http:// revistamexicanadeinvestigacionenpsicologia.com/ojs/index.php/RMIP/ article/download/188/101 ISSN-impresa: 2007-0926; ISSN-digital: 2007-3240

Tamayo, J., Ribes., E.\& Padilla, M. A. (2010). Análisis de la escritura como modalidad lingüística. Acta Comportamentalia, 18, 87-106. Recuperado de: $h$ ttp://www.redalyc.org/pdf/2745/274519450004. pdfISSN: 0188-8145
Truscott, J. \&Hsu, A. (2008). Error correction, revision, and learning. Journal of Second Language Writing, 17, 292-305.Recuperado de http://dx.doi. org/10.1016/j.js/w.2008.05.003ISSN: 1060-3743

Valenzuela, O. (2006). Bioestadística aplicada. Hermosillo: Universidad de Sonora.

Zimmerman, B.\&Kitsantas, A. (2002). Acquiring writing revision and selfregulatory skill through observation and emulation. Journal of Educational Psychology, 94, 660-668.Recuperado de http://dx.doi. org/10.1037/0022-0663.94.4.660 DOI: 10.1037/0022-0663.94.4.660 